\title{
System for Assessing Classroom Attention
}

\author{
Mirko Raca \\ CRAFT \\ Ecole Polytechnique Federale de Lausanne \\ RLC D1 740, Station 20 \\ Lausanne, Switzerland \\ mirko.raca@epfl.ch
}

\author{
Pierre Dillenbourg \\ CRAFT \\ Ecole Polytechnique Federale de Lausanne \\ RLC D1 740, Station 20 \\ Lausanne, Switzerland \\ pierre.dillenbourg@epfl.ch
}

\begin{abstract}
In this paper we give a preview of our system for automatically evaluating attention in the classroom. We demonstrate our current behaviour metrics and preliminary observations on how they reflect the reactions of people to the given lecture. We also introduce foundations of our hypothesis on peripheral awareness of students during lectures.
\end{abstract}

\section{Categories and Subject Descriptors}

K.3.1 [Computer Uses in Education]: Computer-assisted instruction (CAI); K.3.m [Computers and Education]: Metrics-performance measures, Miscellaneous

\section{General Terms}

Measurement, Design, Orchestration

\section{Keywords}

Attention, classroom orchestration, computer vision, behavioural observation

\section{INTRODUCTION}

Learning analytics $(L A)$ started off from the need to govern educational decisions in an informed way[5][9]. Many existing papers give advice on how to conduct each of the five stages of LA (capture, report, predict, act, refine) [14] by taking information systems such as CMS (Class Management System) and SIS (Student Information Systems) as the base of approach.

In this paper we explore possibilities of implementing more unintrusive means for assessing the progress of learners, in environment without digitally quantified inputs. We turn to the most basic (and most common) learning scenario - a teacher in a classroom.

The aim is to develop a system which can monitor attention in the classroom during the lecture and indicate to the lecturer drops in concentration. This will allow us to find

Permission to make digital or hard copies of all or part of this work for personal or classroom use is granted without fee provided that copies are not made or distributed for profit or commercial advantage and that copies bear this notice and the full citation on the first page. To copy otherwise, to republish, to post on servers or to redistribute to lists, requires prior specific permission and/or a fee.

Copyright 2013 ACM 978-1-4503-1785-6/13/04 ...\$15.00. parts of the lecture which were not received with high attention by the audience. In order to have minimal overhead on the existing learning process, we are reducing our inputs to video observations of the students in the classroom. To conserve their privacy, we do not try to connect the images to the identity of the student nor to we try to track a person outside of a single lecture. The goal is not to provide reports on individual students but to give an overall picture of the classroom attention during the lecture.

As the work of Campbell and Oblinger [14] noticed, our intervention needs to be well timed in order for the teaching staff to correct their approach in accordance with the observations of any analytical system. For this reason, our end goal has two possibilities: a real-time reporting system which acts as the indicator of current attention of the students in the class, and summary report of each class which can be presented to the teacher after a finished period of teaching.

In this short paper we introduce our preliminary results and design concept in order to start a discussion with the community. By following the five steps of learning analytics, we will present our methodology for collecting data; our tools for visualization; our preliminary results on reporting and analysis; and ideas for developing the model and presentation of data.

\section{THEORETICAL BACKGROUND}

In the teaching community there is a growing interest in classroom orchestration $([7][4][8][18][6][10])$ as a theory on how to conduct the classroom learning process. The main instrument of action is the teacher [7]. The our work is focused on the factors of awareness and control of what is going on in the classroom, in order to allow the teacher to organize the learning period to the maximum benefit of the listeners.

It is easy for a lecturer to neglect the reception of the topic by the audience, even more so for a novice one. A good teacher has the flexibility to react upon the reception and adjust the course of lecture as part of a "reflection in action" [16].

With this work, we are taking the existing teacher-centric approach and expanding it with our observations about students. There are propositions on how long can the students maintain attention in a given lecture [19]. Our assumption at the moment is that even though classroom learning is not a group activity, there are lateral connections between the participants by which they are affecting each other. We take some concepts from the theory of group work. For instance it 


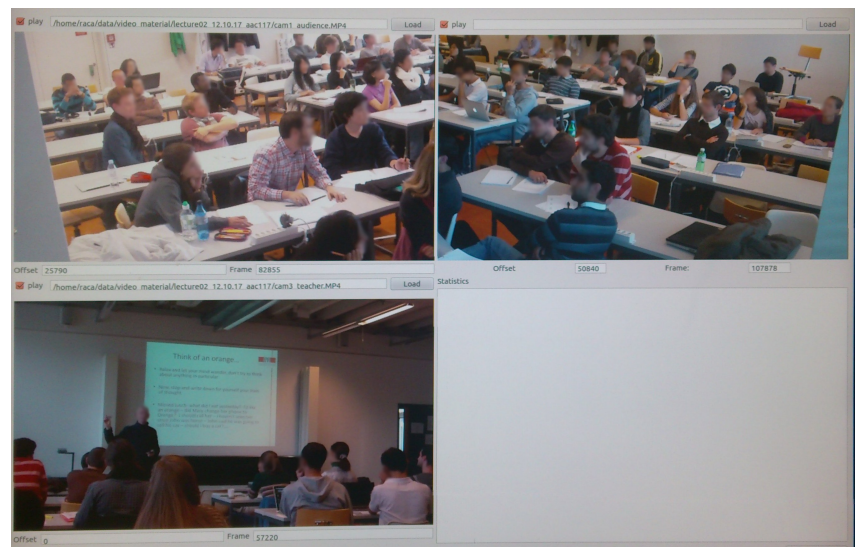

Figure 1: Snapshot of the tool for video synchronisation, annotation and a sample of our recording of a class

is noticed in [15] that contribution of non-conforming group members is perceived as of higher importance then the work of conforming members. We tend to see similar behaviour in reaction to non-conforming audience members paying attention and we are interested to see how this influences other participants. We aim to capture spatial propagations of attention or distraction among students, which we currently call "distraction ripples". The idea is that even though the teacher is the dominant influence, students are not isolated from their surrounding. Our approach can be considered as a counterpart to the research conducted on teacher's movement [13] which we already see as a big influence on attention of students [7]. We have also seen how peripheral awareness can be used to improve classroom interaction [4], and we aim to achieve similar reactions with less intrusive input methods.

\section{STATE OF RESEARCH}

After establishing the theoretical base of our research we present our activities, tools and cues for analysing classroom attention. We are classifying our activities in accordance with the five steps of learning analytics [14].

\subsection{Capture}

Our main source of data are captured videos of classrooms. This is consistent with our determination of making a minimal impact on the classroom existing ecosystem, since the needed modification consists of inserting observational cameras, which are a passive way of collecting data and require no interaction on the subjects side. We are motivated to take this approach with the maturing of computer vision technologies [11] and gradual demystification of human perception [12].

Our setup for collecting data consists of a system of 3 to 4 cameras, of which one is observing the lecturer and the others are used for capturing audience reactions. On Figure 1 we see one of the recordings in the video synchronisation and annotation tool. This allows us to collect, for every recorded lecture, events such as changes of slides, their duration, amount of questions-answers, annotate person locations in the classroom and other properties.

In addition to this, on a limited number of classes, we also
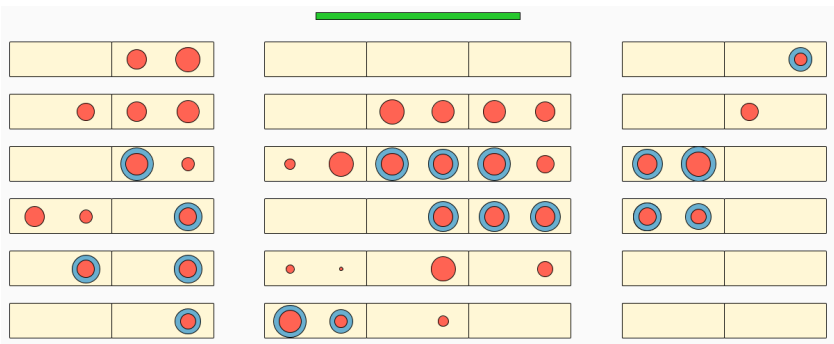

Figure 2: Spatial display of activities in class. Top represents the front of the class. Size of red circles shows reported level of attention. The blue rings indicate subjects that marked that they took notes during that period of the lecture.

collect self-reported levels of attention and actions by means of questionnaires. At four moments during the class, the students are asked to stop and report their level of attention and activities they were doing during the period, whether positive or negative. As noted in Section 2, we take interest into spatial distribution of student actions, and note the location of each subject in the classroom. A sample of our data and visualization can be seen on Figure 2 .

In our visualisation tool, we are able to choose a set of attributes that we want to display (Figure 2), in order to explore how the activities are changing over space and time (at each of the 4 different interruptions). The reason why we did not use one of the existing tools such as Gephi [2] or Cytoscape [1] is that we have a predetermined layout of the graph, with small number of nodes and big variability of display styles which we would like to dynamically change during a presentation. For this reason, we hope that our solution, based on Processing library [3], will provide better visualisations for the intended domain, while it has no intention to compete with the above mentioned tools for other purposes.

\subsection{Report}

In the exploratory phase of the research we are testing different visual indicators of attention during the lecture. We are currently focused on two aspects - quantifying body motion and estimation of gaze direction.

Gaze direction has proven to be high importance cue for human interaction, as it is extensively written in Chapter 6 of [12]. For our usage, we can not assume that we will have high enough resolution of data to estimate the precise point of observation. We are aiming to reach rough estimation, and separation of 3 distinct directions: $i$ ) the teacher/slides, ii) notebook/bench and iii) other directions, which should be reachable even given the low resolution of current input if we take into consideration position of the entire head, similar to $[17]$.

We tested our motion metrics by annotating regions in which each student resides and measuring the amount of movement inside of it. Directions of movement in the nine measured sub-regions for each student can be seen on Figure 3. The current approach is based on estimating optical flow between 2 images by usage of iterative Lucas-Kanade method [20], which we found to be a good combination of speed and robustness.

We find it encouraging that even without localizing the 


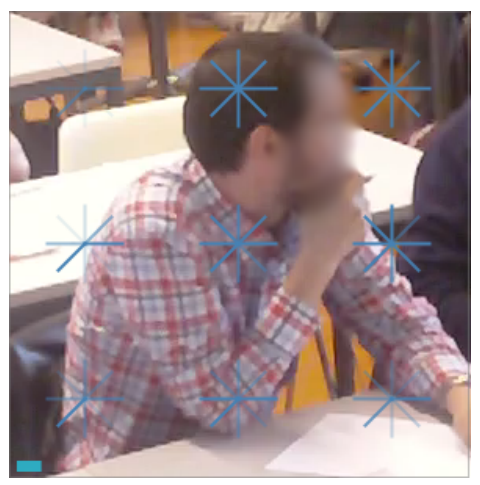

Figure 3: Motion detected of sub-regions of a single person. Blue lines which are more visible indicate higher amount of movement in that region. Orientation of the lines indicate the direction of the movement.

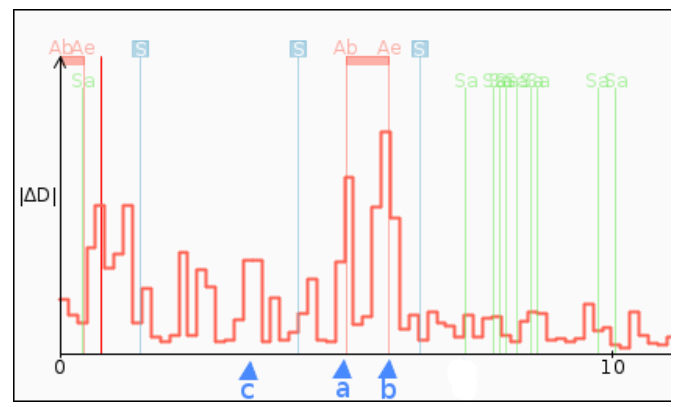

Figure 4: Movement of a single person; at the top of the timeline we display annotated events during the class (slide changes (blue), periods of answering questions (red), slide animations (green)). The red curve represents the normalised amount of movement in intervals of 10 seconds. The amount of movement can allow us to tell the difference between changes in pose/major body movement $(a, b)$ and writing activity (c)

movement (in which sub-section the motion was detected), we can estimate the difference between body-posture shifts, writing action and being still. We try to show importance of these measures and correlation with events during the class by overlaying our annotations of classroom events in Figure 4 .

We would like to note that these visualisations are for primary purpose of evaluating whether our selected cues have the basic potential to provide us with meaningful informations about students activity. Represented in this form, data can become overwhelming, as demonstrated in Figure 5. Each student has an individual set of habits, motions and postures, a set of attributes which renders the data unusable for a simple statistical processing, if we consider isolated individuals.

\subsection{Predict}

We start from the idea that the students are reacting either to a stimulus which is coming from the teacher, or they are reacting to movement of their peers (mimicking attentive behaviour). The assumption is that the class that is paying attention will have higher synchronisation in actions, e.g. higher probability that they will start writing down important information when they hear it. A classroom with low attention would either $i$ ) stay passive or $i i$ ) react in a more sporadic manner. The idea of this kind of measurement is attractive since it's independent of the topic and individual's behaviour.

We can already see higher coordination in activities at Figure 5 at the four points of the class when the students were asked to fill out the attention form (the four periods of filling out the questionnaire are marked with red lines at the top of the time-line). The rest of the time, students tend to behave in an uncorrelated fashion, depending on their personal level of attention.

Another goal for prediction is to use machine learning techniques to process the data of individual students. We have already made the setup for collecting data which can be used for supervised machine-learning, by handing out the questionnaires. The amount of data needed for creating a valuable training set is too big for the current phase of the project, so we set this as our potential long-term goal.

\subsection{Act}

In accordance with the principles of classroom orchestration [7], the acting upon the information is left to the lecturer. Two stages of our process will encourage "reflection on action" by presenting an digest analysis of the classrooms attention to the teacher after the class and "reflection in action" with a indicator of current attention level to the teacher.

As it is seen in previous work [4] the display of information during the class must be kept in a minimal level in order not to become too distracting. With this in mind, an indication to the professor on the amount of attention in the previous 5 minutes would suffice as an signal whether a concept needs to be repeated or the pace of the lecture needs to be changed. We do not intend to provide concrete advices on how to change the lecture.

\subsection{Refine}

As stated in the previous section, our main goal is to see refinement in the mental model of the teacher. As we are in the exploratory phase of the project, our metrics and technologies will pass trough several iterations before we can conclude their relevance in the final framework.

\section{CONCLUSIONS}

We presented our current state of research. Our cues for analysing the attention during the lecture seem promising, and the goal of this paper is to discuss the feasibility of the approach with a broader community. Our immediate goal is to continue the work on the technical "pipeline" of the process in order to increase robustness and quality of the final result.

In the time of massive exploitation of virtual learning environments and new learning concepts, we find that there is still room for improvement of the typical learning scenario. New vision technologies are enabling us to provide relevant statistical analysis of learning situations in a unobtrusive way, and provide the teachers with tools to deliver better lectures on the spot. The task is more challenging with the given variety of scenarios, but we think that by 

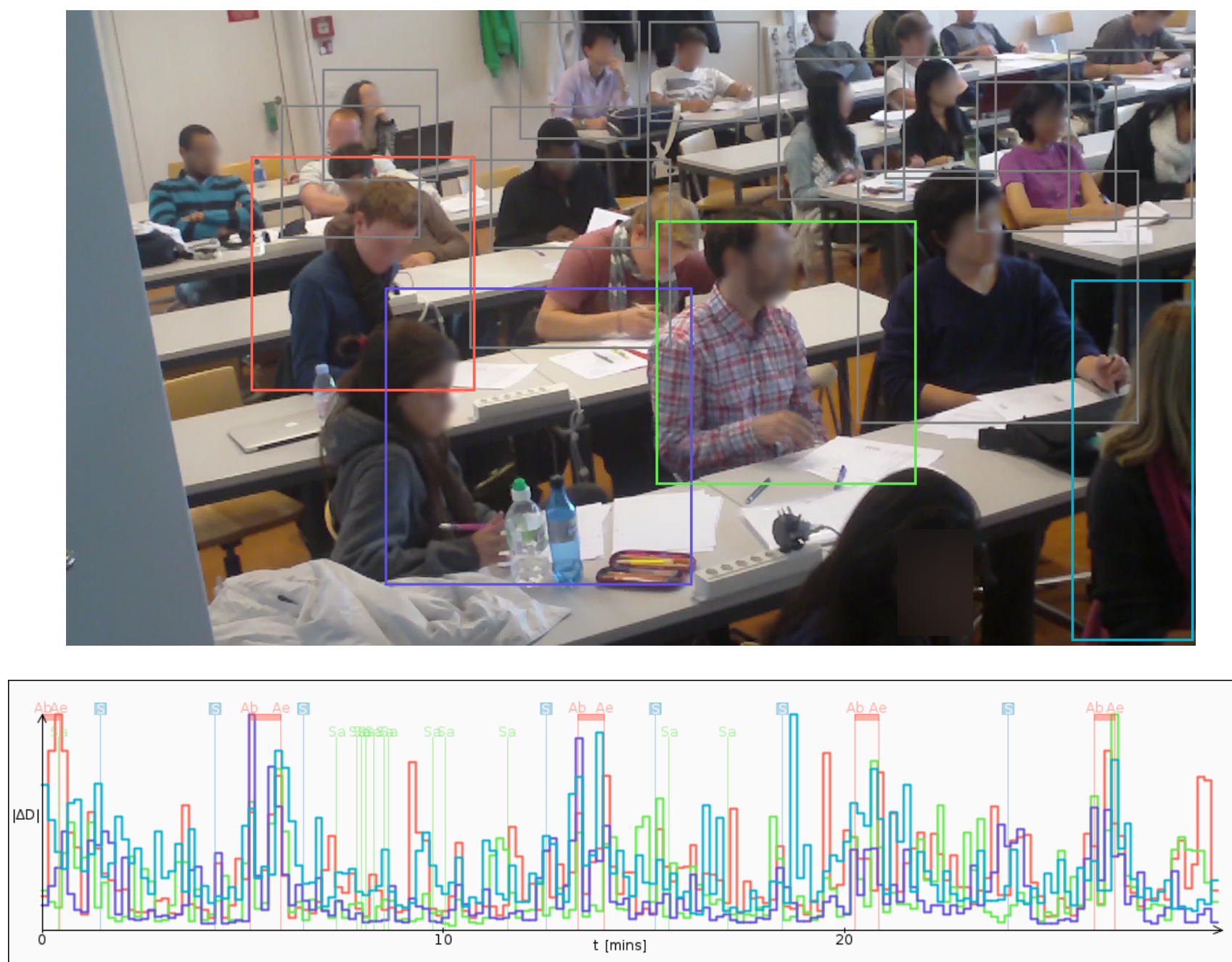

Figure 5: Movement analysis of several persons and associated video with color-coded markers

setting modest goals and using low-demanding technologies we could produce significant benefit for the practise of everyday lecture.

\section{ACKNOWLEDGMENTS}

This work has been sponsored by the ProDoc SNF Grant, project PDFMP1 135108. We would also like to thank all the participants of our experiments.

\section{References}

[1] Cytoscape - an open source platform for complex network analysis and visualization, http://www.cytoscape.org/.

[2] Gephi - open graph viz platform, https://gephi.org/.

[3] Processing, http://processing.org/.

[4] H. S. Alavi, P. Dillenbourg, and F. Kaplan. Distributed awareness for class orchestration. In Learning In The Synergy Of Multiple Disciplines, Proceedings, pages 211-225. 4th European Conference on Technology Enhanced Learning, 2009.
[5] J. Campbell, P. DeBlois, and D. Oblinger. Academic analytics: A new tool for a new era. Educause Review, 42(4):40, 2007.

[6] C. DiGiano and C. Patton. Orchestrating handhelds in the classroom with sris classsync(tm). Computer Support for Collaborative Learning, pages 706-707, 2002.

[7] P. Dillenbourg and P. Jermann. Technology for classroom orchestration. New Science of Learning, pages 525-552, 2010.

[8] P. Dillenbourg, G. Zufferey, H. Alavi, P. Jermann, S. Do-Lenhand, Q. Bonnard, S. Cuendet, and F. Kaplan. Classroom orchestration: The third circle of usability. In International Conference on Computer Supported Collaborative Learning Proceedings, pages 510517. 9th International Conference on Computer Supported Collaborative Learning, 2011.

[9] T. Elias. Learning analytics: Definitions, processes and potential. Retrieved February, 9:2012, 2011.

[10] F. Fischer, C. Wecker, J. Schrader, P. Gerjets, and F. W. Hesse. Use-inspired basic research on the or- 
chestration of cognition, instruction and technology in the classroom. EARLI, 2005.

[11] D. A. Forsyth and J. Ponce. Computer Vision: A Modern Approach. Prentice Hall, 2011.

[12] R. B. A. Jr, N. Ambady, K. Nakayama, and S. Shimojo. The Science of Social Vision. Oxford Series in Visual Cognition, 2010.

[13] F. Lim, K. L. O'Halloran, and A. Podlasov. Spatial pedagogy: mapping meanings in the use of classroom space. pages 235-251, May 2012.

[14] D. Oblinger and J. Campbell. Academic analytics, educause white paper. Retrieved October, 20:2011, 2007.

[15] C. Ridgeway. Conformity, group-oriented motivation and status attainment in small groups. Social psychology, pages 175-188, 1978.

[16] D. Schon. The reflective practitioner: How professionals think in action, volume 5126. Basic Books, 1984.

[17] R. Stiefelhagen. Tracking focus of attention in meetings. In Multimodal Interfaces, 2002. Proceedings. Fourth IEEE International Conference on, pages 273280. IEEE, 2002.

[18] C. A. Tomlinson. The Differentiated Classroom: Responding to the Needs of All Learner. Pearson, 1999.

[19] K. Wilson and J. H. Korn. Attention during lectures: Beyond ten minutes. Teaching of Psychology, pages 8589, December 2007.

[20] J.-Y. Bouguet. Pyramidal Implementation of the Affine Lucas-Kanade Feature Tracker Description of the algorithm Inter corp. 2010 